peaked with the chorea, and disappeared before movements ceased. The mean total duration of chorea symtoms was 7 months. (Swedo SE et al. Sydenham's chorea: Physical and psychological symptoms of St Vitus dance. Pediatrics April 1993; 91: 706-713). (Reprints: Susan E Swedo MD, Child Psychiatry Branch, National Institute of Mental Health, Bldg 10, Room 6N240, 9000 Rockville Pike, Bethesda, MD 20892).

COMMENT. Of 9 children exhibiting obsessive-compulsive symtoms, 4 met DSM-III-R diagnostic criteria for OCD. The authors hypothesize that certain acute-onset cases of OCD acquired during childhood may represent an autoimmune cerebral disorder similar to Sydenham's chorea, and affecting the basal ganglia. A trial of intravenous immunoglobulin in children with Sydenham's chorea is in progress at the NIH.

\title{
LYME DISEASE: PROGNOSIS WITH EARLY TREATMENT
}

The long-term outcome of 63 children with erythema migrans, treated early with antibiotics at the Wildwood Pediatrics Clinic, Essex, Connecticut, was determined by telephone interview 1 to 6 years after the initial episode of Lyme disease. Penicillin V (60\% of patients), amoxicillin (25\%), tetracycline (10\%), or doxycycline (5\%), had been given orally for 10 to 30 days. None of the patients had carditis, arthritis, or neurologic complications attributable to Lyme disease. A recurrence of erythema migrans was reported in 7 (11\%). (Salazar JC et al. Long-term outcome of Lyme disease in children given early treatment. LPediatr April 1993; 122: 591-593). (Reprints: Michael A Gerber MD, Department of Pediatrics, University of Connecticut Health Center, 263 Farmington Ave, Farmington, CT 06030).

COMMENT. Treatment of Lyme disease at an early stage appears to protect from serious neurologic and other sequelae.

A prospective study of the clinical and epidemiological features of 187 consecutive patients with neuroborreliosis recognized in Denmark over a 6-year period showed that $61 \%$ had Bannwarth's syndrome with paresis, a painful lymphocytic meningoradiculitis, during the second stage of the disease. CNS involvement in the early stages was rare; $4 \%$ had myelitis and 1 patient had acute encephalitis. The final morbidity after a 3 year median follow-up was low; disabling sequelae were reported in 9 (5\%) patients. (Hansen K, Lebech A-M. Brain 1992; 115 : 399-423). Measurement of intrathecal anti-Borrelia burgdorferi antibody is a reliable indicator of CNS infection. (Halperin JJ et al. Neurology 1991; 41: 1571). 